Heart 2004;90:818-824. doi: 10.1136/hrt.2003.025049

$\mathrm{F}$ or most haemodynamically relevant heart valve lesions, surgical therapy remains the treatment of choice. It has been consistently shown to provide long lasting relief of symptoms, and its superiority over medical treatment in this regard has been well established. However, in patients undergoing the most prevalent type of heart valve surgery, prosthetic valve replacement, survival rate analysis late after treatment has revealed an impaired prognosis (10 year survival rates of $65 \%$ for aortic valve replacement, $55 \%$ for mitral and combined valve replacement) in all but a minority. ${ }^{12}$ This comprises patients older than 65 years undergoing aortic valve replacement for aortic stenosis, in whom survival relative to an age and sex matched population is normalised following the first postoperative year. It is similarly well known that late mortality is greater in patients undergoing replacement of the mitral than the aortic valve, and for regurgitant versus stenotic valvar lesions. Thus, prognosis after valve replacement is predominantly reflected by the underlying disease with its preoperative condition of the myocardium as well as the state of the coronary circulation. Consequently, the course after valve replacement may be determined decisively by early recognition of significant valvar lesions, in particular, valve insufficiency. In turn, improved follow up can be achieved by timely selection of the appropriate surgical therapy - that is, mitral valve reconstruction instead of replacement in the case of valve prolapse with severe regurgitation. ${ }^{3}$ Furthermore, "good management" of prosthetic heart valves starts preoperatively with the choice of the right valve-for example, a bioprosthesis, thus avoiding the risks of oral anticoagulation in patients requiring aortic valve replacement $\geqslant 65$ years (without atrial fibrillation, severe left ventricular dysfunction, previous thromboembolism, hypercoagulable state), or in patients undergoing mitral valve replacement $>70$ years. ${ }^{4-6}$ Finally, and in the majority of patients with prosthetic valves, postoperative management aiming at an enhanced prognosis consists of controlling antithrombotic and antibiotic medication, of performing the first and subsequent follow up visits, and in a minority, of promptly recognising potentially devastating complications.

\title{
MANAGEMENT OF PATIENTS WITH PROSTHETIC VALVES
}

\section{Antithrombotic treatment}

All patients with a mechanical valve prosthesis require anticoagulant treatment using warfarin or coumadin. Current guidelines recommend a treatment intensity at an international normalised ratio (INR) of 2.0-3.0 for bileaflet or Medtronic Hall valves in the aortic position (after the first three postoperative months), and of 2.5-3.5 in the vast majority of other situations including disk valves, Starr Edwards valves, mechanical prostheses in mitral position, the presence of risk factors for thrombosis (see below), or the first three postoperative months following bioprosthetic valve replacement. ${ }^{5}$ Patients with a biological valve and risk factors for thrombosis, such as atrial fibrillation, severe left ventricular dysfunction (ejection fraction $<0.30$ ), previous thromboembolism, or hypercoagulable state, should continue to be treated by anticoagulation, aiming at an INR of 2.0-3.0. There is evidence that in most cases (except for those patients intolerant of aspirin), the addition of acetyl salicylic acid (ASA) $75-100 \mathrm{mg}$ daily to anticoagulation is beneficial in reducing the risk of thromboembolism, with only a small increased risk of bleeding. ${ }^{7}$ When ASA cannot be used in high risk patients (that is, in the presence of the aforementioned factors), INR may be adjusted to 3.5-4.5.

In patients who experience an embolic event during adequate antithrombotic treatment, anticoagulant treatment should be increased to achieve an INR of 2.5-3.5 (if previous INR was 2.0-3.0) or even 3.5-4.5 (if previous INR was 2.5-3.5). ASA may have to be added or increased in dosage. ${ }^{5}$

Excessive anticoagulation is managed by withholding warfarin or coumadin, by administering vitamin $\mathrm{K}$ or, in the case of bleeding, by the use of fresh frozen plasma.

Haemorrhagic and thrombotic complications are a major source of morbidity and mortality, and therefore may substantially influence the long term course of the disease following prosthetic 
valve replacement. Overall, the risk of bleeding outweighs that of prosthetic valve thrombosis with obstruction (fig l), thromboembolism, or peripheral ischaemic complications. ${ }^{8}$ Thrombosis occurs in 1-3\% of cases annually with thromboembolic events in $0.7 \%$ of cases; the annual risk of bleeding is equal to $2.7 \%{ }^{8}$ Mortality is higher in patients with bleeding complications from anticoagulant treatment ( $0.3 \%$ per year) compared with patients with thromboembolic complications $(0.03 \%$ per year). These complications may have as much to do with the increasing age of the patient as with the valve prosthesis itself. Patients over the age of 70 years have an increased risk of bleeding as do patients recently anticoagulated or when anticoagulation is started. ${ }^{9}$ Risk factors for prosthetic valve thrombosis are periods of insufficient anticoagulation, the position of the mitral valve as compared with aortic valve (relative risk of mitral position twice as high), low cardiac output, the presence of atrial fibrillation, atrial thrombus, previous embolic episodes, and hypercoagulable states including pregnancy. The incidence of thromboembolic events with ball cage, tilting disk, and bileaflet prostheses is estimated to be $2.5 \%, 0.7 \%$, and $0.5 \%$ per year, respectively. ${ }^{2}$

Prosthetic valve obstruction may be caused by either valve thrombosis or pannus ingrowth. In the latter case thrombolytic treatment is ineffective, and the valve needs to be replaced. This implies that thrombolytic treatment is a viable option in patients with thrombotically obstructed valve prosthesis which is, however, not correct in $12-18 \%$ of patients; the acute mortality rate in this situation is $6 \% .^{5}{ }^{10}$ Risk of thromboembolism during thrombolysis with streptokinase, tissue plasminogen activator, or urokinase is also high and amounts to $12 \%$; stroke $3-10 \%$; major bleeding events 5\%; minor, non-disabling bleeding events $14 \%$; recurrent thrombosis $11 \% .^{5}$ Patients with prosthetic heart valve thrombosis who are in unstable haemodynamic condition (New York Heart Association (NYHA) functional class III-IV) should undergo reoperation. Thrombolytic treatment is reserved for patients who are haemodynamically stable.

The duration of thrombolytic treatment depends on the resolution of the obstruction, which is best assessed by transoesophageal echocardiography (TOE) yielding transvalvar velocity gradients (that is, pressure gradients by the simplified Bernoulli equation) and two dimensional and colour Doppler images of the prosthetic valve opening (fig 1). Thrombolysis should be stopped at 24 hours if no haemodynamic improvement is detected or after 72 hours even if it is incomplete. It was recently reported that a first dose of thrombolysis was successful in only $53 \%$ of cases, but repeated sessions were able to augment the rate of success to $88 \%{ }^{10}$ Using a single lytic infusion, success was achieved in $40 \%$ of the obstructive thrombi and in $75 \%$ of the nonobstructive thrombi. Rapid (three hours) and slow (1524 hours) infusion of streptokinase resulted in similar success rates, whereas major complications (3/32 patients) occurred only in the rapid infusion group. ${ }^{10}$

If thrombolysis is successful, it ought to be followed by a combination of heparin until INR values of 3-4 are achieved in aortic valve prostheses and 3.5-4.5 for mitral valve prostheses. ${ }^{5}$ If partially successful, thrombolysis may be followed by subcutaneous heparin (twice daily) plus warfarin or coumadin (INR 2.5-3.5) for three months. The latter may also serve as an alternative to thrombolysis in patients who are haemodynamically stable. Furthermore, American
College of Cardiology/American Heart Association guidelines recommend short term intravenous heparin treatment in patients who are in NYHA class I and II, or a continuous infusion of thrombolytic treatment over several days. ${ }^{5}$ This multitude of different therapeutic regimens for the treatment of prosthetic valve thrombosis reflects the rarity of the problem $\left(0.5-8 \%\right.$ of left sided mechanical prostheses $\left.{ }^{10}\right)$, coupled with a lack of large scale, randomised controlled trials, which would be impractical.

\section{Management of prosthetic valves during pregnancy}

The management of women with prosthetic heart valves during pregnancy poses a particular challenge as there are no available controlled clinical trials to provide guidelines for antithrombotic treatment. Oral anticoagulants cause fetal embryopathy. Subcutaneous administration of heparin is ineffective in preventing thromboembolic events. A systematic review of the literature to determine pooled estimates of fetal and maternal risks has found that the use of oral anticoagulants throughout pregnancy is associated with embryopathy in $6.4 \%$ of live births. ${ }^{11}$ The substitution of heparin between week 6 and 12 of pregnancy eliminated this risk. The risk of fetal wastage was similar in the two mentioned groups. Overall, maternal mortality was $2.9 \%$, and major bleeding events occurred in $2.5 \%$. The regimen associated with the lowest frequency of valve thrombosis (3.9\%) was oral anticoagulation throughout pregnancy, the risk of which substantially increased to $9.2 \%$ under heparin during the first trimester.

Current guidelines recommend that the decision whether to use heparin during the first trimester or to continue oral anticoagulation throughout pregnancy should be made after full discussion of the aforementioned facts with the patient and her partner. ${ }^{5}$ Women with a history of thromboembolism or an older generation mechanical mitral prosthesis who choose not to take oral anticoagulation during the first trimester should receive continuous unfractionated heparin intravenously. Oral anticoagulation should be stopped no later than week 36 and heparin substituted. If labour begins during oral anticoagulation, a caesarean section ought to be performed. ${ }^{5}$ In the absence of bleeding, heparin can be resumed 4-6 hours after delivery and oral anticoagulation begun.

\section{FOLLOW UP OF PATIENTS WITH PROSTHETIC VALVES First postoperative follow up visit}

Patients undergoing cardiac valve replacement should undergo Doppler echocardiography before hospital discharge. Echocardiographic evaluation at baseline is crucial, since it serves as a reference for subsequent examinations. In this context, knowledge about regional and global left and right ventricular systolic function and size, diastolic left ventricular function (not assessable with mitral valve prosthesis), atrial size, function of the native valves, estimates of pulmonary artery pressure, and, above all, the pressure gradient across the newly implanted prosthesis together with determination of the effective valvar orifice area $\left(\mathrm{ERO}, \mathrm{cm}^{2}\right)$, as well as the presence of paravalvar leaks, is useful. ERO is measured using the continuity equation, whereby, in the case of aortic valve prosthesis, the following parameters may be obtained: mean gradient across the valve (by CW Doppler); left ventricular outflow tract diameter and velocity time integral (by PW Doppler); the product of velocity time integral times 

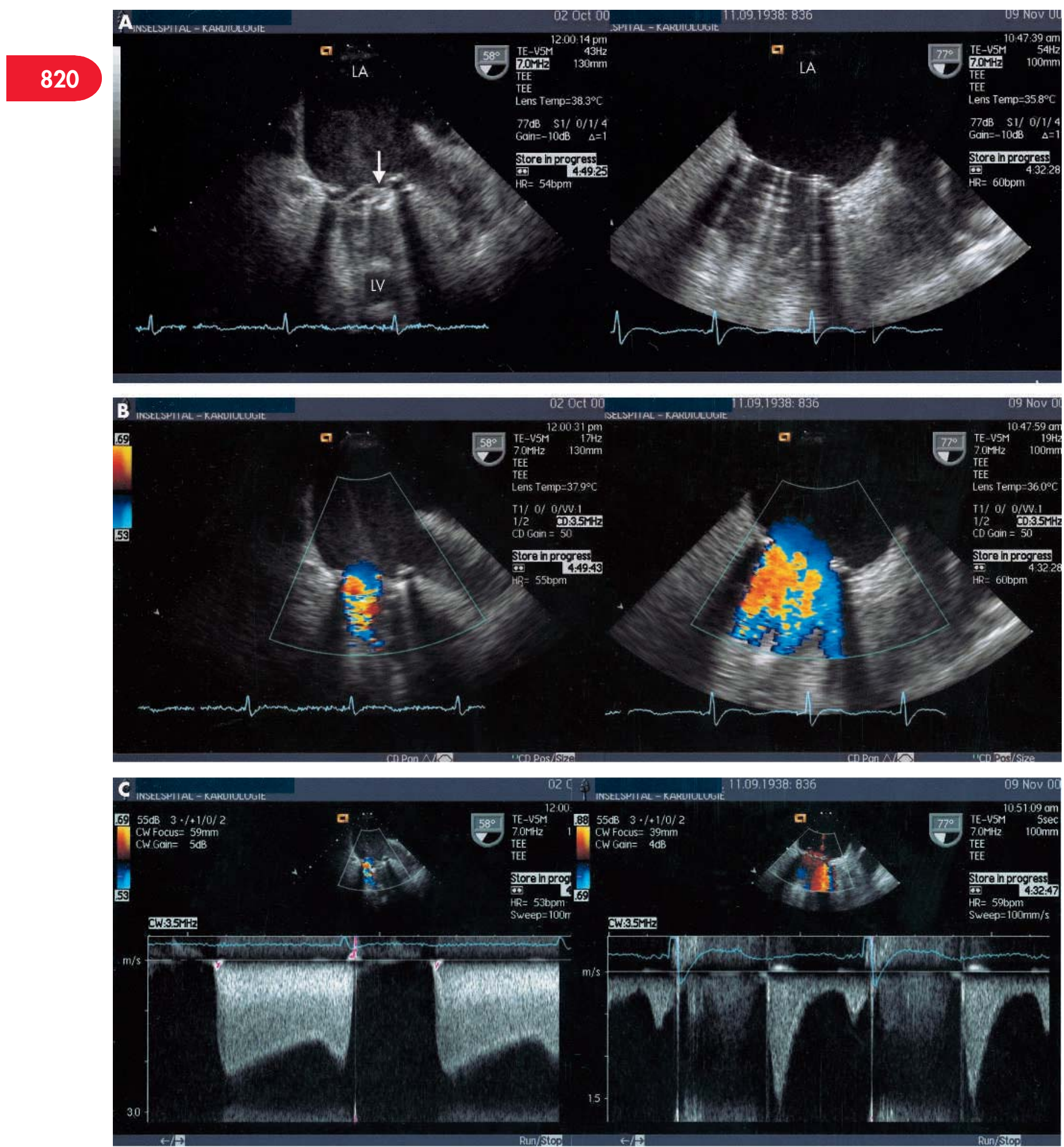

Figure 1 Thrombosis of a mitral valve prosthesis. Panel A depicts a transoesophageal two dimensional image (longitudinal section) of the left atrium (LA) and left ventricle (LV) of a patient suffering a thrombotic obstruction of a bileaflet mitral valve prosthesis (Carbomedics 31). Before thrombolysis (left side), the anterior prosthetic leaflet was completely immobile (arrow), and the posterior leaflet partially immobile. After thrombolysis (right side), both leaflets opened. Panel B illustrates the same situation in the same patient using colour Doppler imaging. The imaging planes are identical to those in panel A. Panel C shows continuous wave Doppler flow velocity spectra across the mitral prosthesis in the same patient before (left) and after (right) thrombolysis. Mean pressure gradient was $14 \mathrm{~mm} \mathrm{Hg}$ before streptokinase treatment and $2 \mathrm{~mm} \mathrm{Hg}$ after. 
Management of prosthetic valves: key points

Management of valve prostheses (particularly in patients with valve regurgitation) begins with selection of the optimal time point for valve replacement

- Aortic prosthesis-patient mismatch (effective orifice area $<0.85 \mathrm{~cm}^{2} / \mathrm{m}^{2}$ ) is prevented by choosing a properly sized prosthesis ring diameter

- Generally, anticoagulation in patients with aortic bileaflet prostheses should be kept at an INR 2.0-3.0; other mechanical valves and bioprostheses three months postoperatively at 2.5-3.5

- Bleeding in patients with valve prostheses is more frequent than valve thrombosis

- Prosthetic valve thrombosis in haemodynamically stable patients should be primarily treated with thrombolytics

- Oral anticoagulation throughout pregnancy is related to a 6-7\% rate of embryopathy

- Intravenous heparin during the first trimester of pregnancy eliminates the risk of embryopathy, but is related to a $9 \%$ risk of valve thrombosis

cross sectional area taken at the same place remains constant (conversation of mass). A body surface area normalised ERO of $<0.85 \mathrm{~cm}^{2} / \mathrm{m}^{2}$ for an aortic valve prosthesis indicates prosthesis-patient mismatch-a term which describes the fact that the prosthesis chosen is too small for the patient's body size. This, of course, should have been prevented during the selection of the valve prosthesis, but is nevertheless important to know for the follow up of the patient, because it may be associated with an increased incidence of morbidity and mortality. ${ }^{12}$ Other reasons for a high transvalvar gradient after aortic valve replacement are septal left ventricular hypertrophy or postoperative high cardiac output. The detection of paravalvar leaks (fig 2) early after operation is important because it alters subsequent follow up (monitoring for haemolysis) and management (possibility of reoperation), and it may be relevant in the diagnostic workup of suspected endocarditis

It is generally recommended that the first outpatient workup should include the patient's history, physical examination, ECG, chest $x$ ray, complete blood count, creatinine, electrolytes, lactase dehydrogenase, INR, and

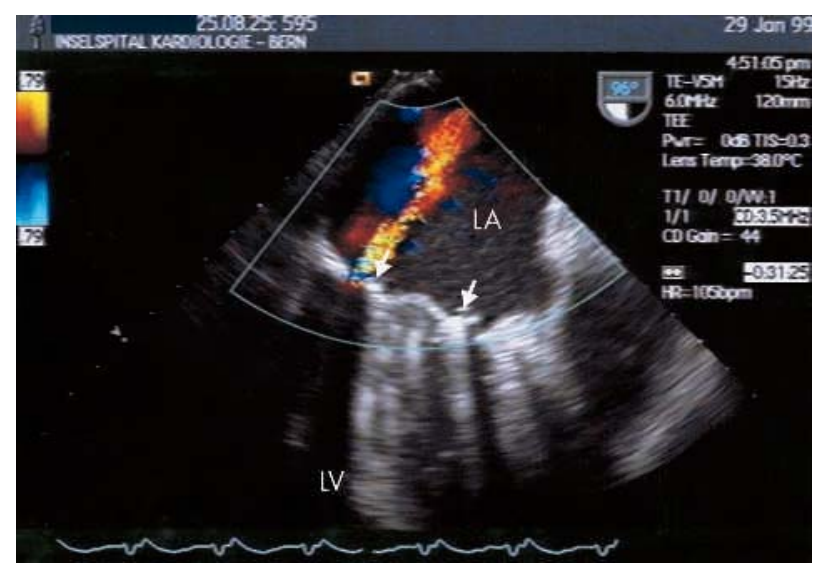

Figure 2 Paravalvar leak. Transoesophageal, two dimensional, colour Doppler image (longitudinal section) of the left atrium (LA) and left ventricle (LV) showing a bileaflet mitral prosthesis with the sewing ring marked by arrows and a posteriorly located paravalvar leak causing mild mitral regurgitation.
Doppler echocardiography. As outlined above, the latter is the most important component of the first postoperative visit because it assesses the effects and results of surgery. ${ }^{5}$

\section{Follow up visits in patients without complications}

Follow up visits in asymptomatic patients without complications and with a "normal" initial echocardiogram can be performed at yearly intervals and should consist of a detailed history taking and a physical examination. There is not much evidence in the literature to support the strategy of performing Doppler echocardiography annually in uncomplicated patients. ${ }^{5}$ Echocardiography is certainly indicated whenever there is evidence of a new heart murmur, when there are questions of prosthetic valve integrity or function, or there are concerns about ventricular function.

\section{Follow up visits in patients with complications}

Any patient with a prosthetic heart valve who does not improve after surgery or who later develops deterioration of functional capacity should undergo appropriate testing including transthoracic or transoesophageal Doppler echocardiography and, if ultrasound is not conclusive, cardiac catheterisation and coronary angiography. Late complications following heart valve surgery can include: thromboembolic and bleeding complications (see above), left and/or right ventricular failure, pulmonary hypertension, sudden death, arrhythmias, conduction abnormalities, mechanical complications, and infective endocarditis (see below). A previous article in this series provides a detailed and comprehensive review of late complications after surgery of valve disease. ${ }^{2}$

\section{Left ventricular dysfunction}

Left ventricular (LV) systolic dysfunction, and consequently also diastolic dysfunction, are most often a consequence of the longstanding preoperative pressure or volume overload. In the case of pure pressure overload as in aortic stenosis, exclusive impairment of LV diastolic function may manifest itself as congestive heart failure after aortic valve replacement. Under the condition that the size of the prosthesis has been chosen to match the patient's body size (see above), LV hypertrophy causing diastolic dysfunction will regress over the course of months and even years. ${ }^{13}$ Doppler echocardiographic assessment of diastolic LV function is challenging because there is an abundance of different velocity parameters obtained during mitral and/or pulmonary vein inflow and at the mitral annulus, which all have to be interpreted in light of the patient's actual preload condition. ${ }^{14}$ Measurement of early diastolic mitral annular motion velocity (normal value $>9 \mathrm{~cm} / \mathrm{s}$ ) is likely the most preload independent Doppler parameter available for LV diastolic function assessment. ${ }^{15}$ LV dysfunction may, alternatively or additionally, be due to the influence of coronary artery disease, poorly controlled systemic hypertension, or coincident cardiomyopathy.

LV systolic function is estimated by measuring ventricular ejection fraction. However, it not only reflects myocardial contractility but is also very susceptible to changes in ventricular pre- and afterload. Altered ventricular loading conditions caused by the valve replacement itself influence the echocardiographically obtained ejection fraction as much as real postoperative changes in myocardial contractility. For example, in the case of severe predominant aortic regurgitation, augmented ventricular preload in the compensated myocardial structure leads to an enhanced contractility 
before valve replacement (Frank Starling mechanism); systolic function will be reduced postoperatively without necessarily reflecting a "poor" outcome following valve replacement. Likewise, a blunted postoperative versus preoperative ejection fraction after mitral valve replacement for severe insufficiency is not by itself an ominous sign, but rather reflects the pathophysiology of a substantial increase in afterload as a consequence of the operation. Conversely, the timing of valve surgery in cases of predominant valvar insufficiency has to account for the pathophysiology of supranormal ejection fraction by choosing still normal values $(<65 \%$ in mitral valve regurgitation and $<55 \%$ in aortic valve regurgitation; $\mathrm{LV}$ end diastolic diameter $\geqslant 45 \mathrm{~mm}$ and $\geqslant 55 \mathrm{~mm}$, respectively) as thresholds for surgery even in the absence of symptoms. ${ }^{3}$

\section{Right ventricular dysfunction}

Haemodynamically relevant tricuspid regurgitation with or without systolic right ventricular (RV) dysfunction is detectable by echocardiography in up to two thirds of patients late after mitral valve replacement, and it is clinically manifest in more than one third. ${ }^{16} \mathrm{RV}$ dysfunction may be the result of persistent LV pathology and the presence of pulmonary artery hypertension, but it may also be caused by uncorrected tricuspid regurgitation with RV volume overload. In this regard, echocardiographic assessment of tricuspid insufficiency and of RV size and systolic function is an integral part of every Doppler echocardiographic examination following valve replacement. Echocardiographic measurement of RV systolic function is inaccurate and insufficiently validated. The latter is true for echo derived RV ejection fraction measurement which is hampered by the crescent-like shape of the RV. A systolic versus diastolic RV area change of less than $20 \%$ is generally regarded as an indicator of reduced systolic RV function. ${ }^{17}$ Measuring (free wall) tricuspid annular motion velocity during systole by tissue Doppler imaging has some potential to become a practical tool for non-invasive assessment of systolic RV function (fig 3; with a motion velocity of $<12 \mathrm{~cm} / \mathrm{s}$ reflecting reduced RV ejection fraction $<50 \%{ }^{18}$ ).

\section{Pulmonary hypertension}

The pathophysiology of pulmonary artery hypertension is illustrated by Ohm's law, PAP $=$ PVR $\times \mathrm{Q}_{\mathrm{p}}+\mathrm{LAP}$, where PAP denotes pulmonary artery pressure, PVR is pulmonary vascular resistance, $Q_{p}$ is pulmonary arterial volume flow rate, and LAP is left atrial pressure. Pulmonary hypertension (mean pulmonary artery pressure $>25 \mathrm{~mm} \mathrm{Hg}$ ) is commonly present in patients with left sided valve disease and is most pronounced in rheumatic mitral valve disease. It is not only the result of elevated left atrial back pressure transmitted across the pulmonary circulation (LAP), but also of an increase in PVR caused by pulmonary arterial vasoconstriction and obliterative changes of the pulmonary vascular bed. Following correction of the left sided valvar lesion, an early fall in pulmonary artery pressure is expected which is the result of a reduction in both PVR and LAP. The detection and monitoring of pulmonary hypertension using Doppler derived systolic velocity gradient measurements between the RV and the right atrium is easy to perform. This is in part due to the fact that tricuspid regurgitation (prerequisite for non-invasive estimation of pulmonary artery pressure) is present in $80 \%$ of the cases with a systolic pulmonary artery pressure above $30 \mathrm{~mm} \mathrm{Hg}{ }^{19}$ The late postoperative persistence or emergence of pulmonary hypertension and right heart failure may reflect delayed left sided valve replacement, new prosthetic dysfunction, or new LV pathology.

\section{Sudden death}

Sudden death as an event complicating the postoperative course of prosthetic heart valve replacement accounts for about $25 \%$ of late deaths after such an operation, and its annual risk is estimated to be $0.2-0.9 \%^{2}$ The majority of sudden deaths are caused by the natural disease process of the valvulopathy, with the most common cause being ventricular arrhythmias. Causes of valve related sudden death include valve thrombosis, thromboembolism, endocarditis, paravalvar leak, mechanical failure of the prosthesis, and intracerebral haemorrhage. In half the cases undergoing aortic valve replacement, atrioventricular conduction problems will finally require permanent pacemaker implantation.

\section{Mechanical complications}

Paravalvar leak (fig 2) in the absence of infection represents one form of mechanical complication with both mechanical and biological valves, and usually reflects suture failure. It may cause significant levels of haemolysis which finally can require reoperation. In mechanical valves, sudden failure of the components of the valve is extremely rare but usually fatal. Gradual deterioration in valve performance caused by slow in-growth of fibrous tissue (pannus) is more frequent, is observed also with bioprostheses, leads to increasing valve obstruction, and can be effectively treated only by reoperation. In biological valves, late degeneration is the major complication. It consists of thinning, atrophy, perforation (in

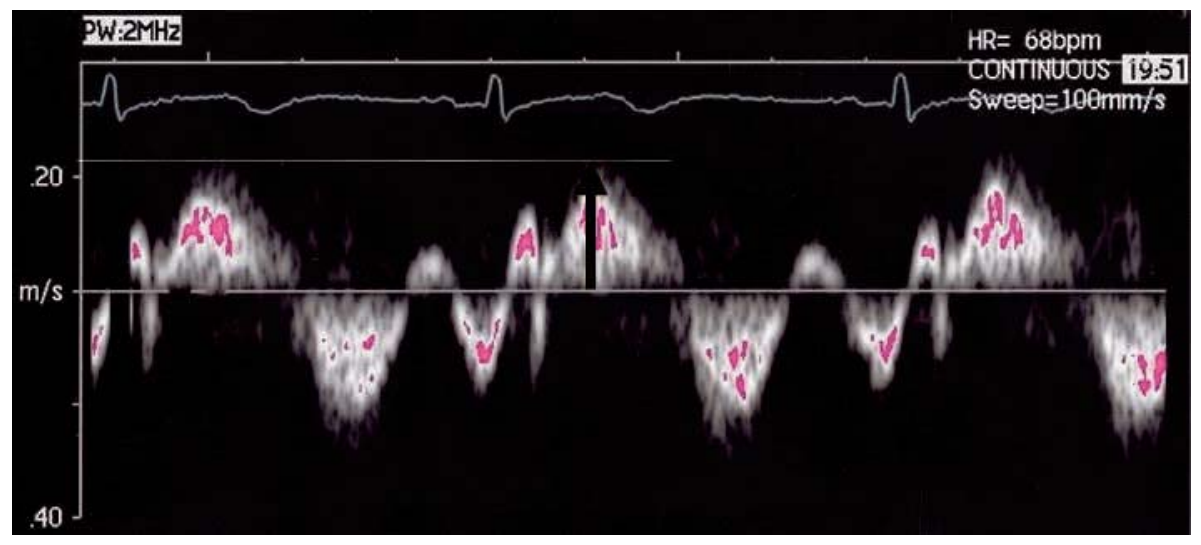

Figure 3 Systolic and diastolic tricuspid annular motion velocity signals. The tissue Doppler imaging signals are obtained using pulsed wave Doppler with the sample volume placed at the free wall tricuspid annulus. A systolic tricuspid annular motion velocity (arrow; in this patient $22 \mathrm{~cm} / \mathrm{s}$ ) of $\geqslant 12 \mathrm{~cm} / \mathrm{s}$ accurately predicts normal systolic right ventricular function. 
Follow up in prosthetic valves: key points

- Immediately after valve surgery, Doppler echocardiography should be obtained in all patients

- The first outpatient workup should include the patient's history, physical examination, ECG, chest $x$ ray, complete blood count, creatinine, electrolytes, lactase dehydrogenase, INR, and Doppler echocardiography

- Follow up visits in asymptomatic patients without complications or new murmurs can be performed at yearly intervals and without Doppler echocardiography

- Complications after valve replacement: thromboembolic bleeding, ventricular failure, pulmonary hypertension, sudden death, arrhythmias, conduction abnormalities, mechanical complications, and infective endocarditis

- Transvalvar or paravalvar regurgitation is detected by Doppler echocardiography; the latter may be harmless, but may represent a haemodynamic problem or may be a sign of prosthetic endocarditis

- Infections of prosthetic heart valves are a very serious complication (0.1-2.3\% per patient year). In suspected endocarditis, the threshold for transoesophageal echocardiography should be low

allografts), cusp thickening, calcification, and tearing. Valve degeneration is accelerated by young age. Rates of aortic valve failure (porcine bioprostheses) over the course of 10 years are reported to be $42 \%$ in patients aged $21-30$ years versus $0 \%$ in those aged $61-70$ years. $^{2}$

\section{Antibiotic prophylaxis and endocarditis}

All patients with prosthetic valves need appropriate antibiotic prophylaxis for the prevention of endocarditis. ${ }^{20}$ As the risk for an infection is much higher in patients with prosthetic heart valves than in those with valvar heart disease, more intensive prophylaxis is needed in the former.
Infective endocarditis is a feared complication following either early (within 60 days of initial surgery) or late after valve surgery, with the infection usually located on the valve prosthesis. Endocarditis in general is a rare condition with a 10 year survival rate of $50 \% .^{21}$ In particular, prosthetic valve endocarditis also occurs infrequently $(0.1-2.3 \%$ per patient year $\left.^{20}\right)$; in our recently reported series of 36 patients with prosthetic valve endocarditis (out of 212 patients with endocarditis), 24 (56\%) died during the course of 7.5 years mean follow up. ${ }^{21}$ Thus, prosthetic as compared to native valve endocarditis was not a risk factor for poor outcome.

Early prosthetic valve endocarditis is caused by contamination of the valve during implantation with culprit organisms such as Staphylococcus epidermidis, Gram negative bacilli, and fungi. In late prosthetic valve endocarditis, streptococci with sites of entry similar to native valve endocarditis are the most common causative organisms. With mechanical prostheses, the infection tends to be located at the sewing ring of the valve, in bioprostheses it can also involve the cusps, while in composite grafts it may even include the distal anastomosis or coronary reimplantation site (fig 4). Vegetations may develop and give rise to systemic embolisation, while periannular tissue destruction may lead to paravalvar leak, and abscess and fistula formation. Clinical presentation and diagnostic criteria are similar to native valve endocarditis. Transoesophageal echocardiography is of particular importance when prosthetic valve endocarditis is suspected because of its high sensitivity to detect small vegetations or abscesses.

Prompt treatment with combined broad spectrum antibiotics is crucial once the diagnosis is suspected and after blood culture sampling has been performed. Parenteral antibiotics should be given over the course of six weeks and modified according to blood culture results following an initial "blind" treatment period. Early surgical intervention as compared to medical treatment alone has been found to be

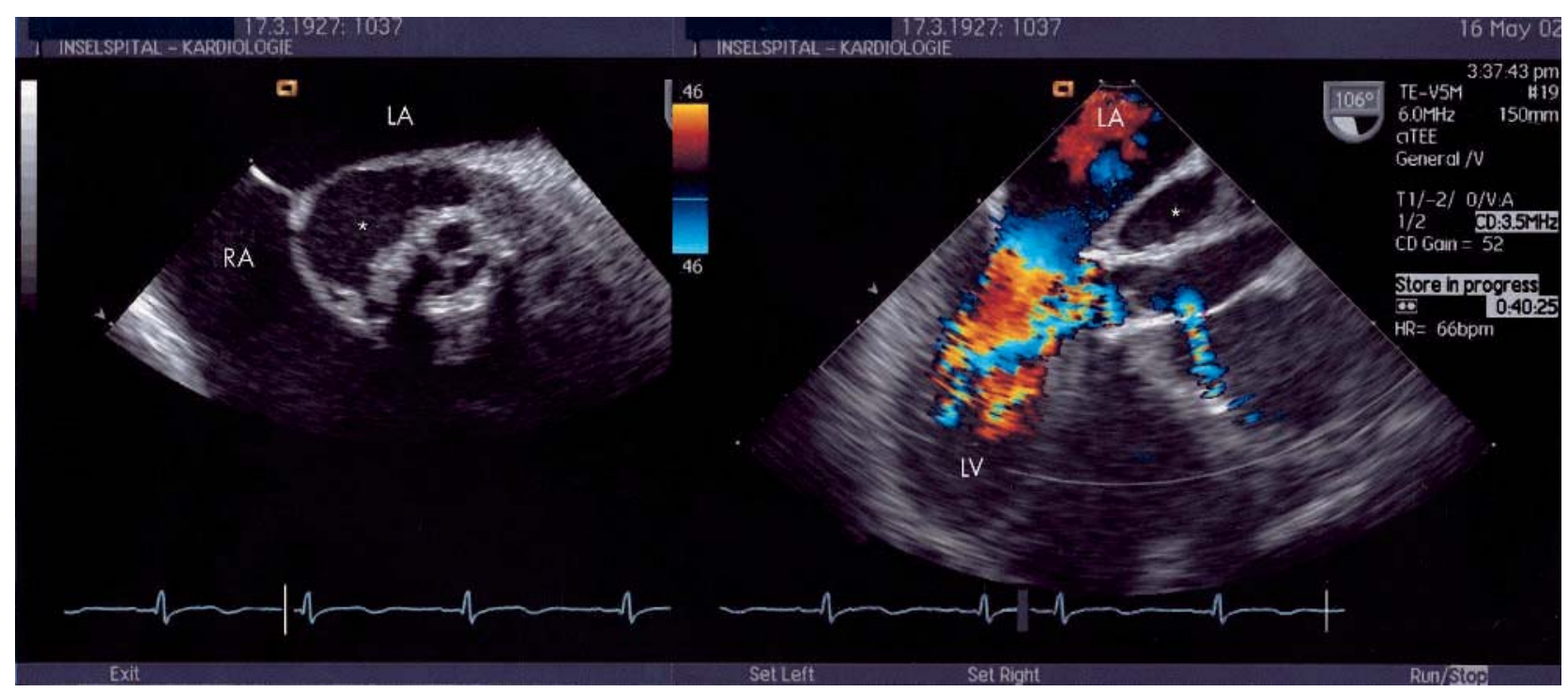

Figure 4 Composite xenograft failure. A transoesophageal two dimensional image in the short axis (left panel) and the long axis projection (right panel, colour Doppler image) shows an extended echo-free space (*) around the aortic composite graft with a bioprosthesis (Shelhigh porcine stentless aortic prosthesis 21). In the short axis view, the left atrium (LA) and right atrium (RA) are depicted as well as the three cusp valve in the centre of the composite graft wall which itself is surrounded by haematoma (*). Both images are obtained during diastole (white lines on the ECG). There is moderately severe aortic insufficiency. Additionally, there is a leak at the right coronary artery reimplantation site. Intraoperatively, a dehiscence was also detected at the distal suture line of the composite graft. Both the distal and the right coronary cusp leakage caused the periaortic haematoma. It was suspected that a low grade endocarditis caused this situation. LV, left ventricle. 
related to better survival. ${ }^{21}$ Since it is impossible to perform controlled clinical trials in endocarditis with randomisation to medical or surgical/medical treatment arms, the benefit of early surgical therapy may reflect a selection bias for surgery in healthier patients. Indications for surgery include prosthesis dysfunction, abscess or fistula formation, systemic multiorgan failure.

For a detailed review of prosthetic valve endocarditis see an earlier article in this series. ${ }^{20}$

\section{REFERENCES}

1 Lindblom D, Lindblom $U$, Qvist J, et al. Long-term relative survival rates after heart valve replacement. J Am Coll Cardiol 1990:15.566-73.

- A large study of survival rates in consecutive patients after heart valve replacement. Through adjustments in background mortality relative survival rates are presented and thereby identify important variables affecting the survival in different valve lesions.

2 Groves P. Surgery of valve disease: late results and late complications. Heart 2001;86:715-21.

3 Enriquez-Sarano M. Timing of mitral valve surgery. Heart 2002;87:79-85

4 Bloomfield P. Choice of heart valve prosthesis. Heart 2002;87:583-9.

5 Bonow RO, Carabello B, de Leon ACJ, et al. ACC/AHA guidelines for the management of patients with valvular heart disease: executive summary. A report of the American College of Cardiology/American Heart Association task force on practice guidelines (committee on management of patients with valvular heart disease). Circulation 1998;98:1949-84.

- A comprehensive review of all aspects of the management of patients with valvar heart disease including valve replacement surgery.

6 Herzog CA, Ma JZ, Collins AJ. Long-term survival of dialysis patients in the United States with prosthetic heart valves. Should ACC/AHA practice guidelines on valve selection be modified? Circulation 2002;105:1336-41.

7 Massel D, Little SH. Risks and benefits of adding anti-platelet therapy to warfarin among patients with prosthetic heart valves: a meta-analysis. J Am Coll Cardiol 2001;37:569-78.

- A meta-analysis of the use of warfarin and aspirin in patients with prosthetic heart valves. It is concluded that combining low dose aspirin with warfarin decreases the risk of systemic embolism or death in patients with prosthetic heart valves.

8 Cannegieter S, Rosendaal F, Wintzen A, et al. Optimal oral anticoagulant therapy in patients with mechanical heart valves. N Engl J Med 1995:333:11-17.

9 Gohlke-Barwolf C. Anticoagulation in valvar heart disease: new aspects and management during non-cardiac surgery. Heart 2000;86:715-21.

10 Oezkan M, Kaymaz C, Kirma C, et al. Intravenous thrombolytic treatment of mechanical prosthetic valve thrombosis: a study using serial transesophageal echocardiography. J Am Coll Cardiol 2000;35:1881-9.

- Useful analysis of the results of intravenous thrombolytic treatment in 32 symptomatic patients with prosthetic valve thrombosis. In comparison to other data in the literature, this single centre cohort is relatively large. Initial success using streptokinase was $53 \%$ as assessed by transoesophageal echocardiography; it increased to $88 \%$ following repeated thrombolytic sessions.

11 Chan WS, Anand S, Ginsberg JS. Anticoagulation of pregnant women with mechanical heart valves: a systematic review of the literature. Arch Intern Med 2000; 160:191-6.

12 Pibarot P, Dumesnil J. Hemodynamic and clinical impact of prosthesis-patient mismatch in the aortic valve position and its prevention. J Am Coll Cardiol 2000;36:1131-41.

- A useful update on the concept of aortic prosthesis-patient mismatch with a review of the present knowledge regarding its impact on haemodynamic status, functional capacity, and patient morbidity and mortality.

13 Mandinov L, Eberli F, Seiler C, et al. Diastolic heart failure. Cardiovasc Res 2000;45:813-25

14 de Marchi S, Bodenmuller M, Lai D, et al. Pulmonary venous flow velocity patterns in 404 individuals without cardiovascular disease. Heart 2001;85:23-9.

15 Nagueh S, Sun H, Kopelen $\mathrm{H}$, et al. Hemodynamic determinants of the mitral annulus diastolic velocities by tissue Doppler. J Am Coll Cardiol 2001;37:278-85.

16 Porter A, Shapira Y, Wurzel $M$, et al. Tricuspid regurgitation late after mitral valve replacement: clinical and echocardiographic evaluation. J Heart Valve Dis 1999;8:57-62

17 Jaffe CC. Echocardiography of the right side of the heart. Cardiology Clinics 1992; 10:41-57.

18 Tüller D, Steiner M, Kabok M, et al. Assessment of systolic right ventricular function by Doppler tissue imaging of the tricuspid annulus [abstract]. Eur Heart J 2002;23:426.

19 Krowka MJ. Pulmonary hypertension. Mayo Clin Proc 2000;75:625-30.

20 Piper C, Körfer R, Horstkotte D. Prosthetic valve endocarditis. Heart 2001;85:590-3.

21 Netzer ROM, Altwegg SC, Zollinger E, et al. Infective endocarditis: determinants of long term outcome. Heart 2002;88:61-6.

- Retrospective cohort study in 212 patients with infective endocarditis followed for an average of 7.4 years. Survival was $50 \%$ after 10 years and was predicted by early surgical treatment, young age, lack of congestive heart failure, and the presence of initial symptoms of endocarditis. 